\title{
3D displacement discontinuity analysis of in-situ stress perturbation near a weak fault
}

\author{
Yutong Chai ${ }^{\oplus *}$, Shunde Yin \\ Department of Civil and Environmental Engineering, University of Waterloo, ON N2L 3G1, Canada
}

Keywords:

Displacement discontinuity

in-situ stress

weak fault

numerical analysis

Cited as:

Chai, Y., Yin, S. 3D displacement

discontinuity analysis of in-situ stress perturbation near a weak fault. Advances in Geo-Energy Research, 2021, 5(3): 286-296, doi: 10.46690/ager.2021.03.05

\begin{abstract}
:
A numerical investigation utilizing the 3D displacement discontinuity method is performed to examine the stress perturbations and induced displacements near a weak fault with arbitrary orientations and dip, assuming zero shear stress and normal displacement. The in-situ stress field near the fault is taken as known and varied with depth. The modelling is constructed based on indirect boundary integral equations. In this work, the fault plane is first modelled as a rectangular plane with negligible thickness between the adjacent surfaces. The fault plane is then divided into numerous rectangular boundary elements with imposed shear singularities on the surface, which is normal to the fault plane to simulate a traction-free scenario. The numerical results of the total induced stresses and displacements are then compared to the existing solutions of a penny-shaped crack for validation purpose. With validated results, the paper moves on to the discussion of various factors that have impacts on the induced stress and displacements, including: aspect ratio which is defined by strike over dip; orientation of the strike on the horizontal ground surface; as well as dip. The boundary integration method with modification is also used to model an elliptical distribution of singularities with inner, corner, and edge elements to accommodate more complex shape of a discontinuity; small differences are observed.
\end{abstract}

\section{Introduction}

Knowledge of in-situ stress is essential in civil, mining and petroleum engineering, and many efforts have been made towards its determination lately that provide new perspectives (Dusseault et al., 1998; Li et al., 2000; Zhang and Yin, 2014; Kaiser et al., 2016; Corkum et al., 2018; Faraji et al., 2021). An in-situ stress field with three principal stresses aligned in vertical and horizontal directions and constant stress gradients is usually assumed to represent the stress condition where faults are absent. However, at regions where a fault zone is in vicinity, the alteration of direction and magnitude of the principal stresses should be expected, which has been analyzed by analytical or numerical methods under various settings and assumptions (Homberg, 1997; Li, 2015; Anyim and Gan, 2020; Wang, 2020). Associated with stress perturbation, locally induced displacements and faulting behavior near fault tips due to the in-situ stress have also been studied intensively (Hazeghian and Soroush, 2017; Shi et al., 2020).

Consideration of perturbed stresses and displacements near a fault is important in mining, geotechnical tunneling and petroleum engineering as well as in earth science when the behaviour of the rocks and soils is sensitive to local stresses. Precise field measurement of local in-situ stress state in a large area is either impractical or can be very costly (Zhao et al., 2018; Kuszewski et al., 2021). Alternative analytical and numerical analysis assuming relatively homogeneous and isotropic strata is typical ( $\mathrm{Li}, 2015)$. Before numerical methods are used more frequently in recent decades, simple analytical solutions of the stress perturbations were derived from the theory of elasticity and the relationship between stress and strain (Jaeger and Cook, 1969; Mandl, 1986; Pollard and Segall, 1987). With the advancement of numerical modelling, perturbation of in-situ stress by more complex settings of discontinuities has been evaluated, including stress perturbation near dipping fault (Hazeghian and Soroush, 2017; Lobatskaya et al., 2018; Wang, 2020), fracture localization along faults with varying friction (Cooke, 1997), undersea geo stress field (Li, 2015), and disturbed stress field near discontinuous faults (Segall and Pollard, 1980). Among these efforts, various

\section{Yandy
Scientific}

Press
${ }^{*}$ Corresponding author.

E-mail address: y5chai@uwaterloo.ca (Y. Chai); shunde.yin@uwaterloo.ca (S. Yin).

2207-9963 (c) The Author(s) 2021.

Received May 4, 2021; revised May 29, 2021; accepted May 29, 2021; available online June 2, 2021. 


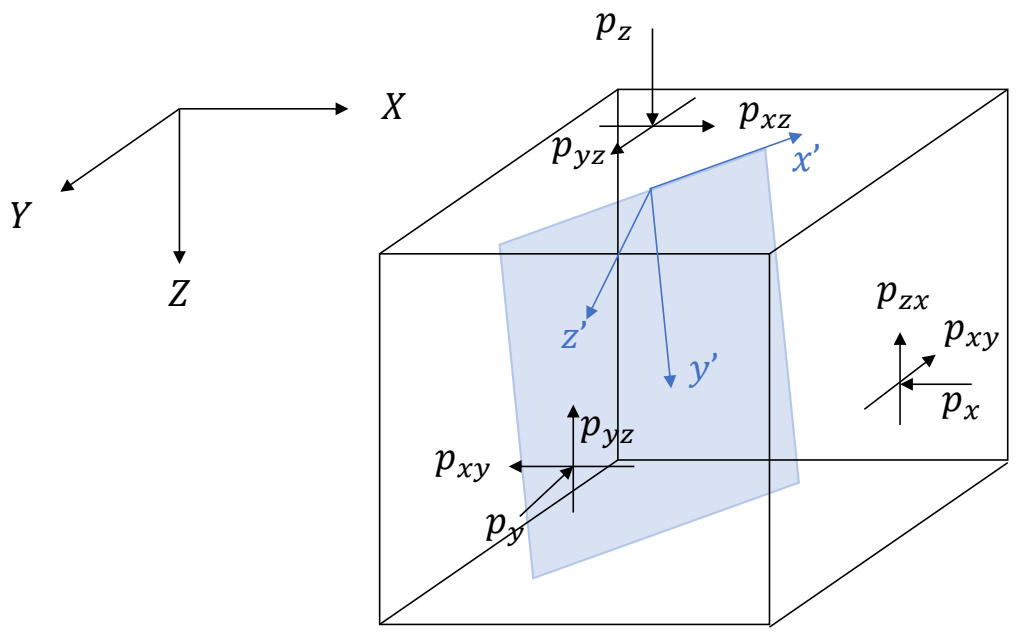

Fig. 1. Model of fault with far-field stresses.

numerical methods have been applied, including finite element method (Henk, 2004; Lobatskaya et al., 2018; Wang, 2020), distinct element method (Homberg et al., 1997; Hazeghian and Soroush, 2017; Gao et al., 2021), displacement discontinuity method (Ritz et al., 2012; Li, 2015), and boundary element method (Brady, 1978; Beer and Poulsen, 1994; Ramos and Daros, 2021).

In this paper, we focus on investigation of the impacts of various parameters influencing the stress perturbation near a weak fault, the most drastic scenario of alteration of the stress field in terms of principal stress orientation. A weak fault is found at a fault region where its shear strength is too low to suppress the applied shear tractions, thus unable to restrict the reorientation of the induced principal stresses (Kuwahara et al., 2021). As an example, the San Andreas Fault in California has been characterized as a weak fault with a shear strength as low as $20 \mathrm{MPa}$. With such low frictional strength of the fault, it shows significant stress rotation (Rybicki, 1995; Fulton et al., 2009). The weakness of a fault can be attributed to several reasons, including earthquakes, decrease of the confining pressure due to near-lithostatic fluid pressure, and pressure solution (Streit, 1997). The decrease of shear stresses may vary between less than 10 to $40 \mathrm{MPa}$ depending on the nature of the earthquake and the fault system itself (Bletery et al., 2017; Zalohar et al., 2020). In order to simulate the most drastic scenario of a weak fault, the coefficient of friction is taken to be zero for all simulations conducted in this paper.

3D displacement discontinuity method (DDM) as a modification of the boundary element method, with proved efficiency in simulating the mechanical behaviours around thin, slitlike openings or cracks in mining and petroleum engineering (Crouch and Starfield, 1983; Fotoohi and Mitri, 1990; Verde and Ghassemi, 2015; Yin et al, 2016; Shen and Shi, 2019), will be employed to carry out numerical experiments on different scenarios of investigations, including the impacts of aspect ratio, dip, strike, and the rectangular/elliptical shaped fault plane.

\section{Methodologies}

The elastic law is the fundamental for the numerical computations of DDM, which expresses induced stresses and displacements at any given point on the interior surface. Consider a model as illustrated in Fig. 1 for a fault with arbitrary strike direction and dip, bounded by domain with a global coordinate system of $(X, Y, Z)$.

Compared to the lateral extent of a fault plane, the thickness is small enough to be considered negligible. Thus, the 3D boundary $S$ becomes two planar surfaces that can be differentiated with subscripts + and - , in an infinite body governed by the global coordinate system. In order to proceed with the numerical analysis using DDM, $S$ is discretized into finite numbers of planar displacement discontinuty (DD) elements. A rectangular DD element has uniform traction and displacement components, while a non-rectangular element has nonuniform distributions (Brady, 1979; Wu and Olson, 2015;). Each element is assigned with a local coordinate system $\left(x^{\prime}, y^{\prime}, z^{\prime}\right)$ depending on its angle of rotation. In addition, each element has two surfaces normal to its local $z^{\prime}$-axis: $S^{\prime+}$ and $S^{\prime}$, as shown in Fig. 2 . The figure depicts the general configuration for a rectangular DD element and its two surfaces, for which the displacement discontinuities along the local coordinate axes are defined as:

$$
\begin{aligned}
& D_{x}=u^{-}-u^{+} \\
& D_{y}=v^{-}-v^{+} \\
& D_{z}=w^{-}-w^{+}
\end{aligned}
$$

where $u, v$ and $w$ are the displacements along each local axis.

This paper will focus on the scenario of a weak fault system without normal displacement, but only shear displacements across the fault plane. Thus $D_{z}$ equals zero; only $D_{x}$ and $D_{y}$ are required to characterize such configuration.

\subsection{Formulation of 3D DDM}

The presence of the shear displacements and zero normal displacement generate shear traction-free surfaces upon the 
local axis of each DD element representing a weak fault. To replicate such scenario, it is necessary to apply a fictitious distribution of shear singularities on each element so that the shear stresses on the plane normal to the local $z^{\prime}$-axis, $\tau_{y z}$ and $\tau_{x z}$, are eliminated. Based on Kelvin Equations, Brady (1979) has derived expressions for stresses and displacements due to such shear singularities, $S_{x}$ and $S_{y}$, as shown in Eq. (2), corresponding to a discontinuity as shown in Fig. 1.

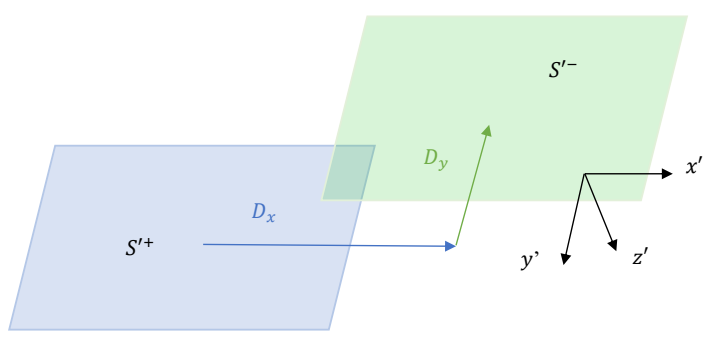

Fig. 2. 3D rectangular DD element.

$$
\begin{aligned}
\sigma_{x} & =\frac{S_{x}}{4 \pi(1-v)} \frac{3 x z}{R^{5}}\left(1-\frac{5 x^{2}}{R^{2}}\right)+\frac{S_{y}}{4 \pi(1-v)} \frac{3 y z}{R^{5}}\left(1-2 v-\frac{5 y^{2}}{R^{2}}\right) \\
\sigma_{y} & =\frac{S_{x}}{4 \pi(1-v)} \frac{3 x z}{R^{5}}\left(1-\frac{5 y^{2}}{R^{2}}\right)+\frac{S_{y}}{4 \pi(1-v)} \frac{3 y z}{R^{5}}\left(1-2 v-\frac{5 y^{2}}{R^{2}}\right) \\
\sigma_{z} & =\frac{S_{x}}{4 \pi(1-v)} \frac{3 x z}{R^{5}}\left(1-\frac{5 z^{2}}{R^{2}}\right)+\frac{S_{y}}{4 \pi(1-v)} \frac{3 y z}{R^{5}}\left(1-\frac{5 z^{2}}{R^{2}}\right) \\
\tau_{x y} & =\frac{S_{x}}{4 \pi(1-v)} \frac{3 y z}{R^{5}}\left(v-\frac{5 x^{2}}{R^{2}}\right)+\frac{S_{y}}{4 \pi(1-v)} \frac{3 x z}{R^{5}}\left(v-\frac{5 y^{2}}{R^{2}}\right) \\
\tau_{x z} & =\frac{S_{x}}{4 \pi(1-v)} \frac{1}{R^{5}}\left(1+v-\frac{3 v y^{2}}{R^{2}}-\frac{15 x^{2} z^{2}}{R^{4}}\right)+\frac{S_{y}}{4 \pi(1-v)} \frac{1}{R^{3}}\left(1+v-\frac{3 v x^{2}}{R^{2}}-\frac{3 y^{2} z^{2}}{R^{4}}\right) \\
\tau_{y z} & =\frac{S_{x}}{4 \pi(1-v)} \frac{3 x y}{R^{5}}\left(v-\frac{5 z^{2}}{R^{2}}\right)+\frac{3 x y}{4 \pi(1-v)}\left(v-\frac{5 z^{2}}{R^{2}}\right) \\
U & =\frac{-S_{x}}{8 \pi G(1-v)} \frac{z}{R^{3}}\left(1-2 v+\frac{3 x^{2}}{R^{2}}\right)+\frac{-S_{y}}{8 \pi G(1-v)} \frac{3 x y z}{R^{5}} \\
V & =\frac{-S_{x}}{8 \pi G(1-v)} \frac{3 x y z}{R^{5}}+\frac{S_{x}}{8 \pi G(1-v)} \frac{z}{R^{3}}\left(-1+2 v-\frac{3 y^{2}}{R^{2}}\right) \\
W & =\frac{-S_{x}}{8 \pi G(1-v)} \frac{x}{R^{3}}\left(1-2 v+\frac{3 z^{2}}{R^{2}}\right)+\frac{S_{y}}{8 \pi G(1-v)} \frac{y}{R^{3}}\left(-1+2 v-\frac{3 z^{2}}{R^{2}}\right)
\end{aligned}
$$

where $v$ is Poisson's ratio, $G$ is modulus of rigidity, and $R$ is defined as:

$$
R=\sqrt{x^{2}+y^{2}+z^{2}}
$$

Using Eqs. (2) and (3), the following resultants can be calculated for each DD element: the magnitudes of three induced normal stress components along the global axes, $\sigma_{x}, \sigma_{y}, \sigma_{z}$; the magnitudes of three induced shear stress components on the global axial planes, $\tau_{x y}, \tau_{x z}, \tau_{y z}$; the three displacement components with respect to the global axes, $U$, $V, W$.

\subsection{Modification for elliptical plane}

Equations given in above section are sufficient to simulate a rectangular fault plane. Now consider an elliptical-shaped penny crack, as shown in Fig. 3, where the corner and edge elements do not fully overlap with the area of each corresponding rectangular DD element.

It is observed that the DDM rectangular elements can be subdivided into three categories as shown in Fig. 4: inner, edge, and corner element.

The inner element, for which local and global axis share the same orientation, does not need further modifications from Eq. (2). For the edge element which is depicted in Fig. 4(b),

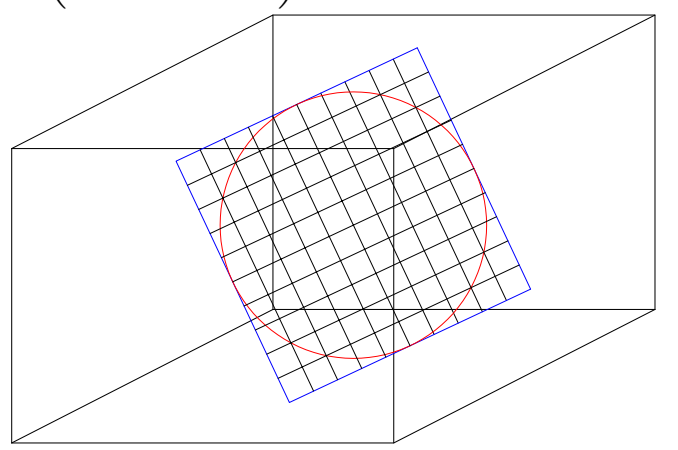

Fig. 3. Illustration of elliptical-shaped penny crack.

shape. The resultant singularity intensity on the edge of the element at point $\left(x_{1}^{\prime}, y_{1}^{\prime}\right), t_{2}$, can be expressed in the following terms derived by Brady (1979):

a quasi-elliptical distribution of the shear singularities is used to approximate the change in intensities due to the elliptical

$$
\begin{aligned}
& t_{2}=t_{1}\left[0.188+\frac{0.812}{x_{2}^{\prime 2}}\left(2 x_{2}^{\prime} x^{\prime}-x^{\prime 2}\right)\right]=t_{1} f_{1}\left(x^{\prime}\right) \\
& 0 \leq x^{\prime} \leq x_{2}^{\prime}
\end{aligned}
$$




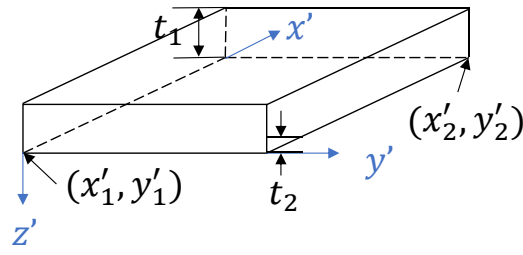

(a) Inner element

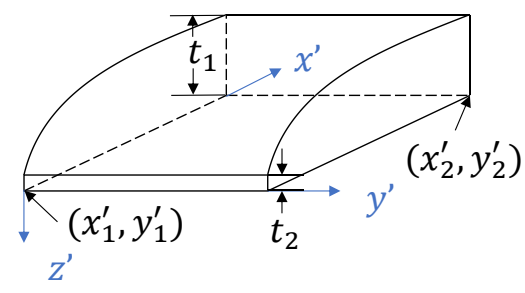

(b) Edge element

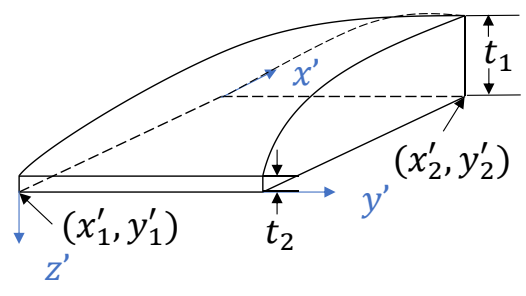

(c) Corner element

Fig. 4. Singularity intensity variations over (a) Inner, (b) Edge, and (c) Corner element.

where $t_{1}$ is shear singularity intensity at point $\left(x_{2}^{\prime}, y_{2}^{\prime}\right) ; x^{\prime}$ is the $x$ coordinate on the center of the element, relative to the local axis.

The above stated distribution induces a stress component, $\sigma$, at any point of interest $i$ with a coordinate of $\left(x_{i}, y_{i}, z_{i}\right)$ on the global axis, which is expressed as:

$$
\sigma=t_{1} \int_{x_{1}^{\prime}}^{x_{2}^{\prime}} \int_{y_{1}^{\prime}}^{y_{2}^{\prime}} f_{1}\left(x^{\prime}\right) f_{2}(x, y, z) d x^{\prime} d y^{\prime}
$$

where

$$
\begin{aligned}
& x=x_{i}-x^{\prime} \\
& y=y_{i}-y^{\prime} \\
& z=z_{i}-z^{\prime}
\end{aligned}
$$

$f_{2}(x, y, z)$ is the expression of induced stress and displacement due to shear singularities that can be found in Eq. (2). The double integral can be solved either analytically or numerically, depending on the desired level of accuracy.

The modification of the quasi-elliptical distribution of shear singularity intensity on an edge segment should be applied to both adjacent edges for a corner segment, as illustrated in Fig. 4(c). To express the elliptical paraboloidal distribution superimposed on a uniform distribution for shear singularity intensity on a corner segment, Brady (1979) has derived the following equation:

$$
\begin{aligned}
t_{2}= & t_{1}\left[0.188+0.812\left(\frac{2+\cos (2 \theta)}{2 x_{2}^{\prime}} x^{\prime}+\frac{2-\cos (2 \theta)}{2 y_{2}^{\prime}} y^{\prime}\right.\right. \\
& \left.\left.-\frac{2+\cos (2 \theta)}{2 x_{2}^{\prime}} x^{\prime 2}-\frac{2-\cos (2 \theta)}{2 y_{2}^{\prime}} y^{\prime 2}+\frac{x^{\prime} y^{\prime}}{x_{2}^{\prime} y_{2}^{\prime}}\right)\right] \\
= & t_{1} f_{3}\left(x^{\prime}, y^{\prime}\right)
\end{aligned}
$$

where $\theta=y_{2}^{\prime} / x_{2}^{\prime}$.

Similar to that composed for the edge element, the stress and displacement components induced by the modified shear singularity intensity $t_{2}$ are obtained by integrating over the area of the corner element with respect to its local is, as below:

$$
\sigma=t_{1} \int_{x_{1}^{\prime}}^{x_{2}^{\prime}} \int_{y_{1}^{\prime}}^{y_{2}^{\prime}} f_{3}\left(x^{\prime}, y^{\prime}\right) f_{2}(x, y, z) d x^{\prime} d y^{\prime}
$$

\subsection{Numerical procedure for DDM}

With the equations derived for the induced stresses and displacements due to a point shear singularity applied to each DD element, and given that the fault plane has been divided into a total of $n$ rectangular elements, the stress transformation took place at an arbitrary point of interest $i$ on the global axis due to the distribution of shear singularities over the fault plane is the sum of stress perturbations on all $n$ elements. As an example, Eq. (7) expresses the relationship as:

$$
\sigma_{x i}=\left[A_{s}\right]\left[S^{\prime}\right]
$$

where $\left[A_{s}\right]$ is the coefficient vector with an order of $[i, n] ; S^{\prime}$ is the vector of shear singularities consisting of distributions on $x z$ and $y z$ planes which can also be written as $\left[S_{x}, S_{y}\right]$, with an order of $[n, 2]$. Same relationship can be found for the rest of five stress components and three displacement components.

An equality is established between the sum of shear stresses at the center of each DD element and the assumedly known far field shear stresses on the same plane. This equality can be expressed in the following terms:

$$
\left[C O E_{s}\right]\left[S^{\prime}\right]=\left[p_{s}\right]
$$

where $\left[C O E_{s}\right]$ is a square matrix of order $2 n$, which can also be written as $\left[\begin{array}{cc}F_{S} & 0 \\ 0 & E_{S}\end{array}\right]$, determined by the same method as stated in Eq. (7); $\left[p_{s}\right]$ is a column vector of known far field stresses, which can also be written as $\left[\begin{array}{l}-p_{x z} \\ -p_{y z}\end{array}\right]$.

The only unknown in the above equation is the shear singularities for each DD element, which can be solved by Gauss-Siedel iteration. MATLAB's built-in matrix solver with proven efficiency and accuracy is utilized in this paper to perform the calculations.

\section{Validation}

Due to lack of analytical solutions, the resulted induced stresses acquired using DDM in this paper are compared to existing sets of numerical simulations for stress distributions evaluated in the same scenarios. The comparable solutions are obtained through a model designed by Brady (1979) simulating a mining excavation around openings in a tabular orebody using boundary element method.

The scenario described by Brady is a square slot in an infinite medium, oriented in the same direction as the global 




Fig. 5. Distribution of shear stress around square slot with $p_{x z}=1$.

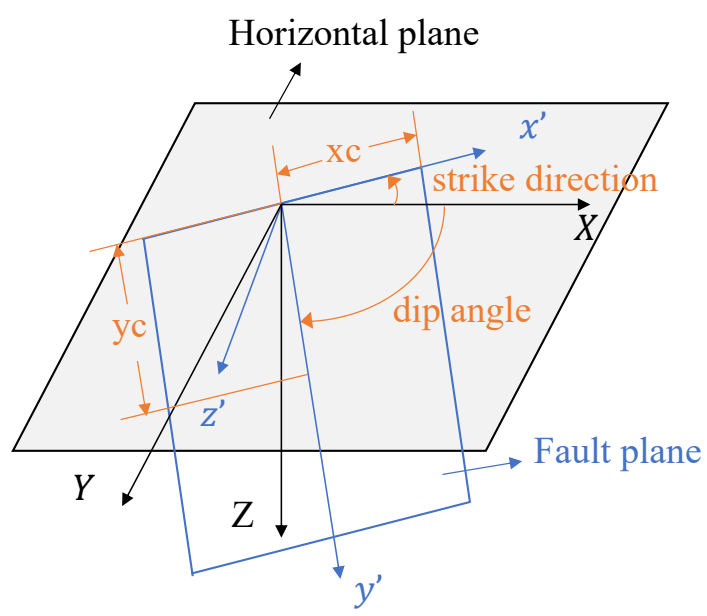

Fig. 6. Simulation configurations for the fault plane.

axis of the fault plane. The far field stress state assigned to the simulation has a magnitude of unity for $p_{x z}$, while the other five stresses are zero. This is similar to the scenario of a zerostrike, zero-dip, squareplane discontinuity on the surface. The variation of induced stress $\tau_{x z}$ is plotted against $X$-axis over half of the length of the fault plane with values of $Y$ and $\mathrm{Z}$ for the observation points be zero, as shown in Fig. 5.

The modulus of rigidity is taken as $40 \mathrm{GPa}$, while the Poisson's ratio is 0.25 . The square fault plane is subdivided into $110 \mathrm{DD}$ elements with equal length and width, which is both computationally efficient and sufficient to represent the entire surface. It is observed that the results from both programs are in good agreement and indicates a satisfactory performance of the developed model for the analysis of induced stresses.

\section{Numerical experiments}

For the following simulations, the configuration presented in Fig. 6 is used. The length of the strike is taken as $2 \mathrm{~km}$, which implies that $x c$, or half-length of the fault strike, is $1 \mathrm{~km}$ in length. The strike direction is measured counterclockwise from the positive $X$-axis, and the dip is measured with respect to the $X Y$ plane, the horizontal plane on the top of the fault. Limestone is chosen to be the sample rock with mechanical properties given by Lama and Vutukuri (1978): the density of the rock is $2700 \mathrm{~kg} / \mathrm{m}^{3}$, with an average Young's modulus of $60 \mathrm{GPa}$ and Poisson's ratio is 0.25 . Both cohesion of the discontinuity and coefficient of the internal friction are considered as zero. The following in-situ stress state evaluated upon the horizontal plane is used to model the fault with respect to its global axis: $80 \mathrm{MPa}$ in $X$-direction, $50 \mathrm{MPa}$ in $Y$-direction, and $60 \mathrm{MPa}$ in $Z$-direction. Increase of in-situ stresses in the far field over depth is described by a gradient of $27.0 \mathrm{kPa} / \mathrm{m}$. The assumed principal stresses correspond to a thrust faulting stress regime of the far field, for which the lowest principal stress is perpendicular to the horizontal plane (Anderson, 1951).

An initial configuration of a zero-strike rectangular fault plane with the strike direction parallel to the global $X$-axis and a dip of $60^{\circ}$ is simulated with the DDM model as the reference case, as shown in Fig. 7. The six contour plots illustrate the spatial distributions of the magnitudes of three induced principal stresses with varying orientations with respect to the global stress field, denoted by sigma 1 , sigma 2 , and sigma 3 ; as well as the three displacement components along the corresponding global axes, $U, V, W$, respectively. The induced principal stress sigma 1 and displacement $U$ both show a pointreflection nature about the midpoint of the fault strike line; rough symmetry can be observed for the remaining induced stresses and displacements about the strike line. Various spatial distributions for the above reference case reveal the difference in results for each comparison that will be presented in the later sections, where the observation points are placed on the sampling line shown as red dotted line in Fig. 7, parallel to the global $Y$-axis with $X$ values equal to zero. In the following sections, series of $1 \mathrm{D}$ plots consisting of the three induced stress components and three displacement components will be generated instead of 2D contour plots. This will help to better visualize the differences between various scenarios by changing the aspect ratio, dip, strike direction, as well as the geometrical configuration of the modelled plane.

\subsection{Impact of aspect ratio of the fault}

The aspect ratio is defined as the ratio of fault's total depth over the length of the strike line; in other words, $y c$ over $x c$ as illustrated in Fig. 6. To examine the impacts of aspect ratio, the simulations of induced principal stresses and displacements using the DDM discussed in this paper are carried out for a rectangular fault plane, as shown in Fig. 8, with the strike direction equal to zero and a dip of $60^{\circ}$ for the following variations of aspect ratios: $0.5,1,2$, and 5 . Due to the presence of singularities, the values between $(-0.6,0.6)$ are excluded.

From Fig. 8, the results show a prominent asymmetry on the hanging wall and the footwall. The reverse in magnitudes along the opposite side of the fault can be interpreted as a reverse in the orientation of the induced principal stresses (Homberg et al., 1996). For observation points located on the sampling line that is perpendicular to the strike line, the deviations for both stresses and displacements from the farfield stresses and zero line, respectively, increase as the aspect ratio becomes larger. As the aspect ratio becomes even greater, 

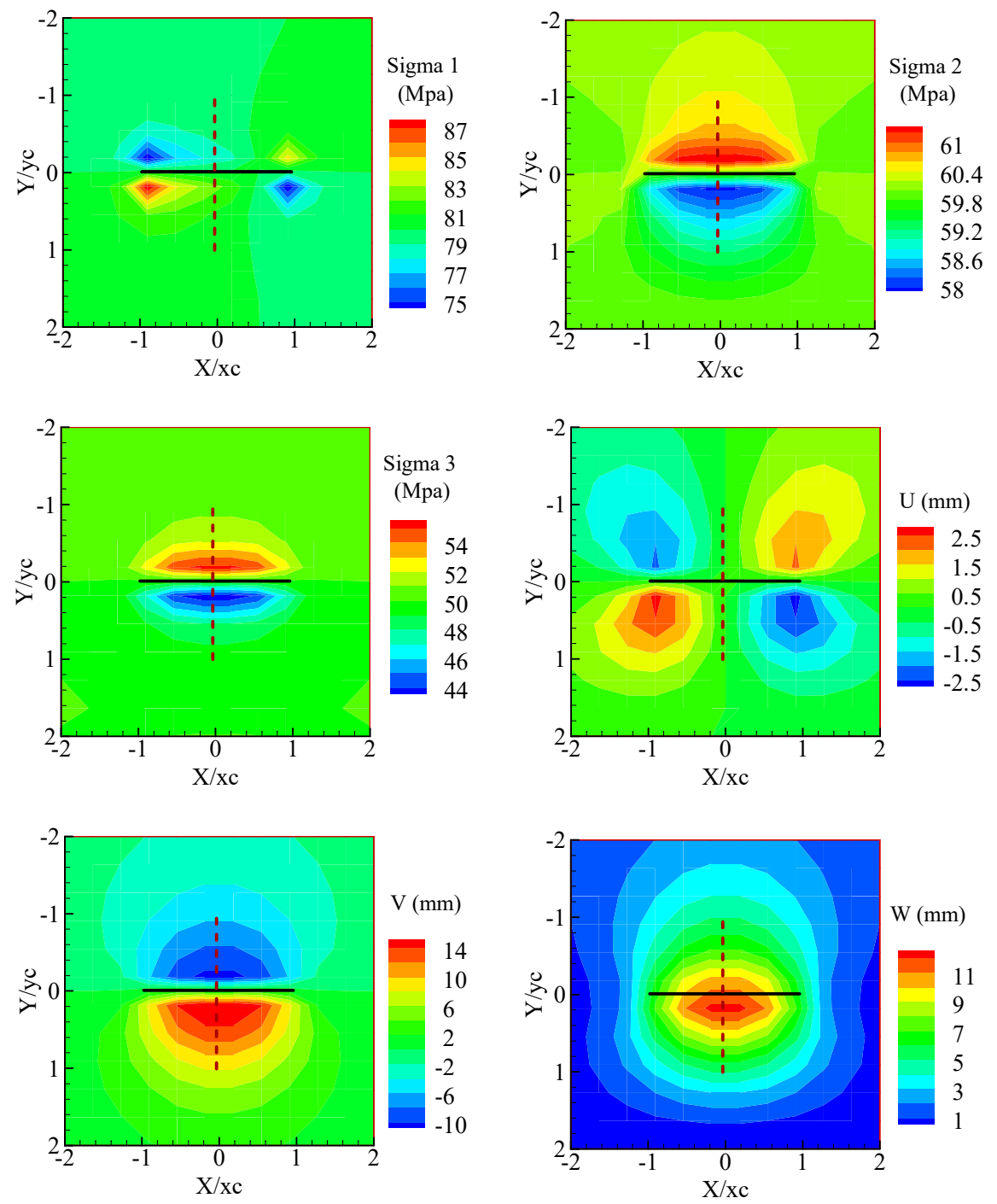

Sampling line

Fault strike line

Fig. 7. Induced stress and displacement contours simulating a zero-strike, rectangular fault plane with a dip of $60^{\circ}$.
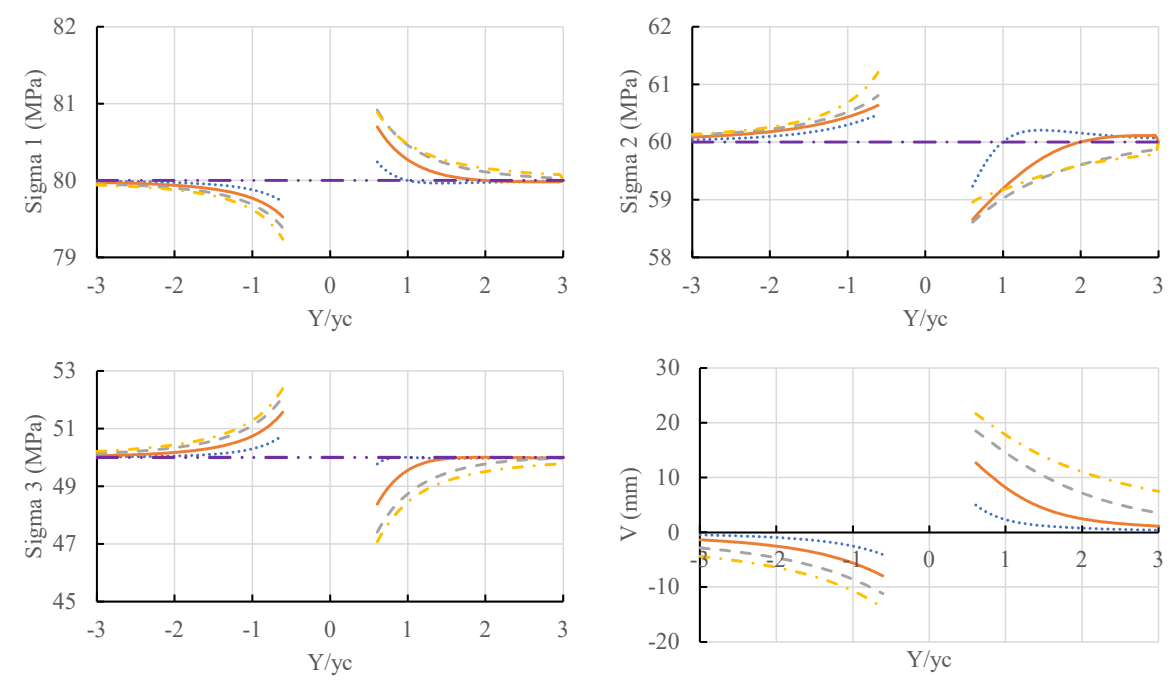

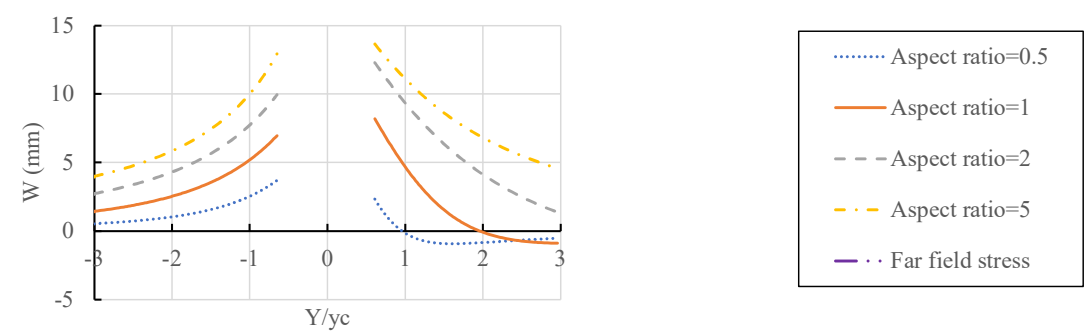

Fig. 8. Induced principal stresses and displacements near rectangular fault plane with varying aspect ratio.
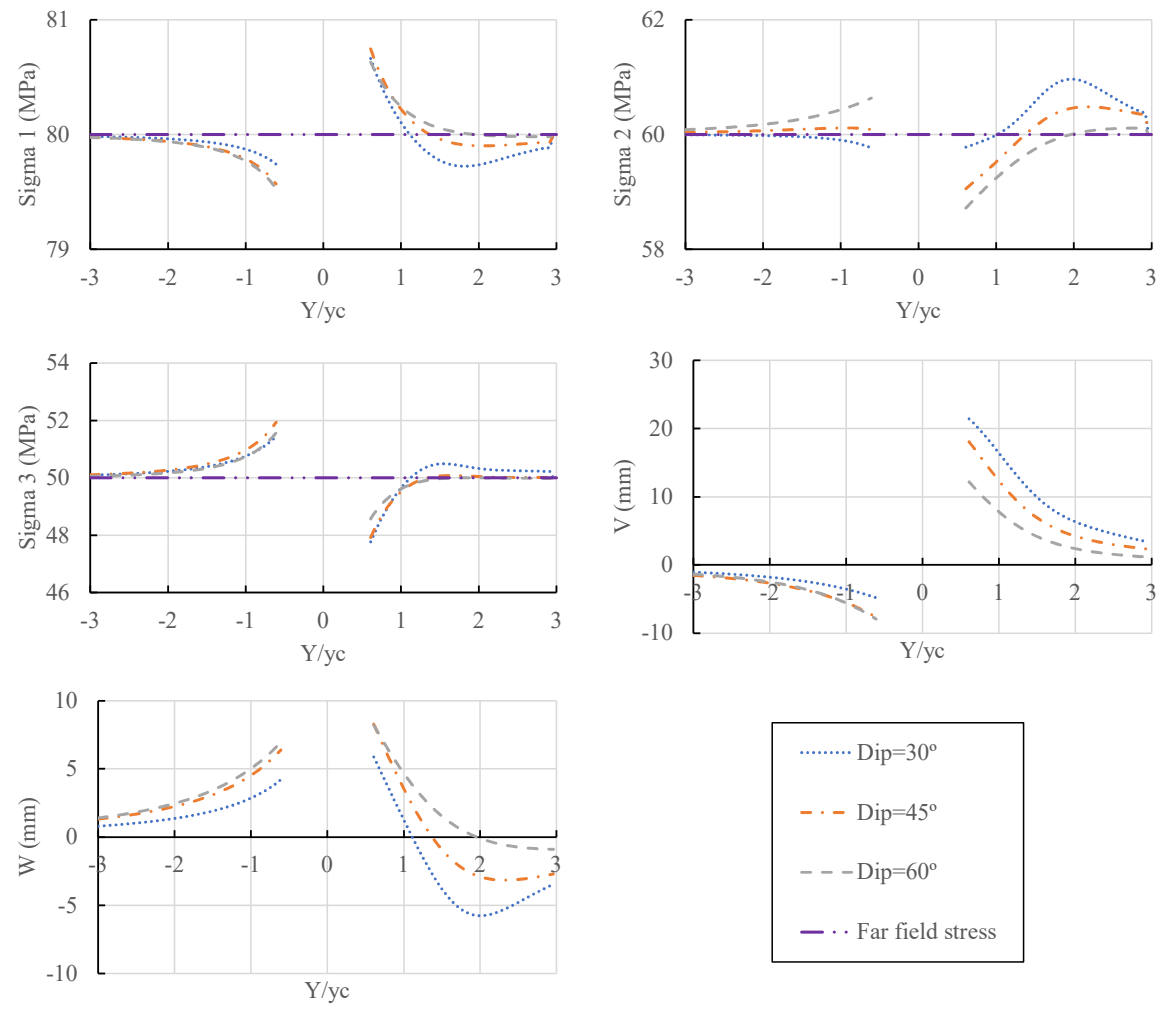

Fig. 9. Induced principal stresses and displacements near a zero-strike, square fault plane with varying dip angles.

with a drastic increase from 2 to 5 shown in Fig. 8, its impact on the induced stresses and displacements largely decreases compared to the cases where the aspect ratio increases from 1 to 2 , or from 0.5 to 1 . It should be noted that this impact cannot be demonstrated in plane-strain assumption based analysis, and therefore cautions should be taken in any 2D analysis of in-situ stress perturbation near a fault.

\subsection{Impact of dip angle of the fault}

The dip of the fault, as illustrated in Fig. 9, is assessed for its impact on the induced stresses and displacements. The simulations are carried out for a zero-strike, square fault plane with the following dip angles: $30^{\circ}, 45^{\circ}, 60^{\circ}, 90^{\circ}$.

As seen in Fig. 9, for a thrust-faulting stress regime of far field, asymmetry is observed for all induced stresses and displacements along the opposite walls of a simulated fault. Despite its impact on each parameter to different extents and even in opposite trends in some cases, the dip angle is clearly influencing the induced stresses and displacements.
Note that for the case of displacement W, where increasing dip angles always led to larger displacements on the same point of observation along the sampling line. It should be noted that such impact of dip angle from a 2D analysis of in-situ stress perturbation near a fault may be derived differently due to the simplification of the stress state.

\subsection{Impact of the strike of a fault}

According to Jaeger and Cook (1969), the pre-existing planes of strike can be reactivated when external stresses are applied at certain directions. Simulations of a square fault plane with a dip of $60^{\circ}$ are conducted for various strike directions that are illustrated in Fig. 10.

The following trends can be observed from Fig. 11, where the induced stresses and displacements due to the presence of a fault with strike directions of $45^{\circ}, 135^{\circ}, 225^{\circ}$, and $315^{\circ}$ are plotted upon the sampling line: fault pairs with strike directions of $135^{\circ}$ and $315^{\circ}$, as well as $45^{\circ}$ and $225^{\circ}$, show prominent symmetry about the midpoint of the strike line 


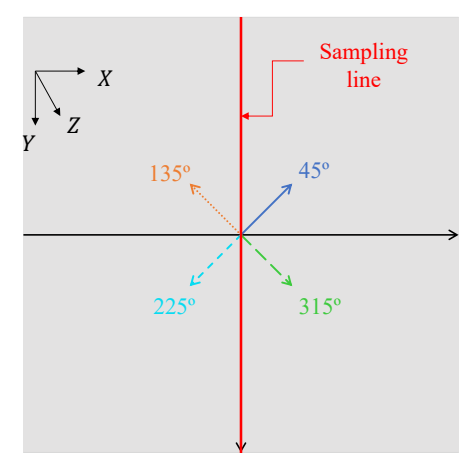

(a)

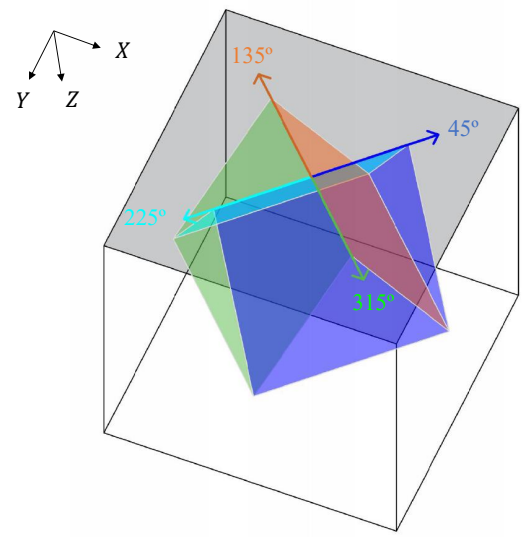

(b)

Fig. 10. Variations of strike direction used for the simulations carried out using a square fault plane with $60^{\circ}$ of dip. (a) $2 \mathrm{D}$ plan view on the plane; (b) $3 \mathrm{D}$ model.
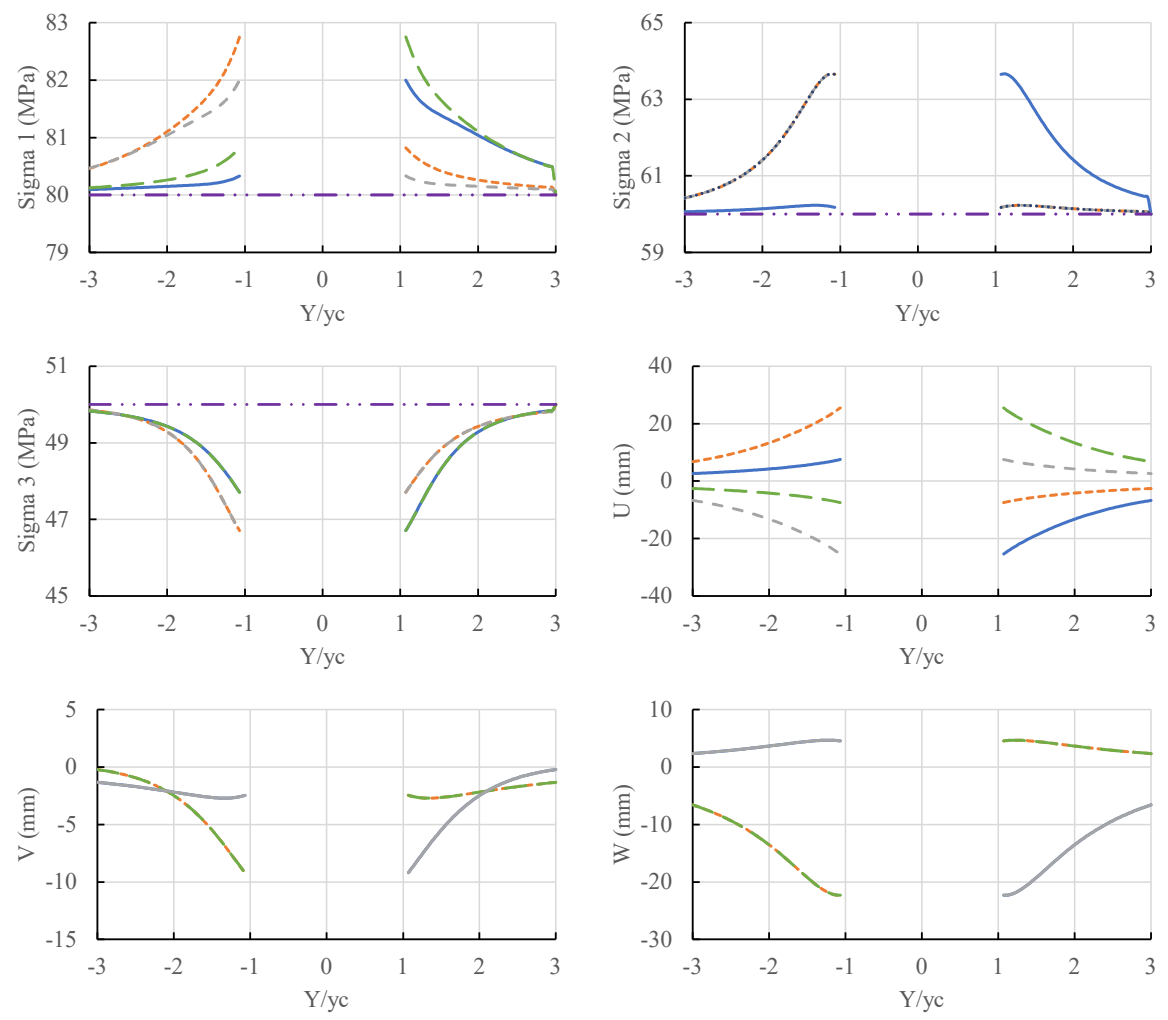

- Strike $=45^{\circ} \cdots$ Strike $=135^{\circ} \cdots$ Strike $=225^{\circ} \quad-\quad$ Strike $=315^{\circ} \quad \cdots$ Far field stress

Fig. 11. Induced principal stresses and displacements near a square fault plane with varying strike direction.

on the opposite sides of the fault, for both induced stresses and displacements. In addition, for sigma 2 and sigma 3, as well as displacements $V$ and $W$, the results are identical for fault pairs with strike directions of $45^{\circ}$ and $225^{\circ}$, as well as $135^{\circ}$ and $315^{\circ}$. This relationship was revealed in Fig. 7 that demonstrated the spatial distributions of in-situ stress state: sigma 1 and $U$ bare point reflection in the center of the strike line, while the rest of the stresses and displacements are symmetrical about the strike line.

\subsection{Impact of the shape of an elliptical fault plane}

As discussed in the previous section, a modification is available for an elliptical discontinuity surface, compared to a rectangular plane that has been used for the previous discussions. A simulation of induced stresses and displacements near a fault with aspect ratio equals unity is conducted for rectangular and elliptical modelling, as shown in Fig. 12. To accommodate the differences drawn from rotating directions of principal stresses, the model is constructed to simulate a 



Fig. 12. Induced principal stresses and displacements near a fault with a dip of $60^{\circ}$.

fault with a dip of $60^{\circ}$ and a strike direction of $0^{\circ}$. The spatial distribution of in-situ stress state for this configuration simulating a rectangular plane is illustrated in Fig. 7.

Similarities are observed for induced principal stresses for observation points on the sampling line; the average differences between the two models are below $0.7 \%$. On the other hand, the induced displacements show significant differences, particularly for the vertical displacement perpendicular to the ground, where the maximum difference is $26.2 \%$. This is noteworthy for monitoring the faulting process in earth science and suggests that the shape of the fault might be taken into consideration. The induced displacements for the rectangular model are larger than the elliptical model in both cases, which is a reasonable conclusion since the area of applied shear singularities for the rectangular fault plane is larger than the elliptical fault plane given the same fault width and depth, as illustrated in Fig. 3.

\section{Conclusion}

In this paper, a 3D DDM is developed to study the impacts of various parameters on the induced stress and displacements near a weak fault zone. With modifications implemented, the model can simulate both rectangular and elliptical fault planes with varying configurations including aspect ratio, dip angle, strike direction, and in-situ stress states before the perturbation. Numerical experiments under a thrust-faulting regime based on the developed model led to the following conclusions:

1) The deeper a fault penetrates in dip direction, or the shorter a fault strike line is compared to its depth, the larger the in-situ stress perturbation will be locally. This impact can be reduced as the aspect ratio increases in magnitude.

2) The dip angle of a fault plane clearly influences stress states and deformations near an fault, without an uniform trend observed.

3) Fault planes that share the same strike line and opposite dipping directions have same degree of perturbations on the opposite side of the fault. Fault pairs with strike directions that are symmetrical by the local $X$-axis bare identical degree of perturbations for its in-situ stress state that has symmetrical spatial distribution.

4) Models based on fault planes with elliptical and rectangular configurations show virtually identical results for induced stresses but much more significant differences for the displacements; induced displacements from the rectangular model always appear to be larger than the elliptical model in terms of magnitudes. The selection of model can be made based on the characterization of the discontinuity geometry.

These findings provide useful references for local in-situ stress perturbation characterization and faulting monitoring in mining, petroleum, and civil engineering as well as in earth science, where results can vary when impacts of aspect ratio, dip and strike angle are not well considered.

\section{Acknowledgement}

Support of the Natural Sciences and Engineering Research Council of Canada (NSERC) is greatly acknowledged. 


\section{Conflict of interest}

The authors declare no competing interest.

Open Access This article is distributed under the terms and conditions of the Creative Commons Attribution (CC BY-NC-ND) license, which permits unrestricted use, distribution, and reproduction in any medium, provided the original work is properly cited.

\section{References}

Anderson, E. M. The Dynamics of Faulting and Dike Formation with Application to Britain. Edinburge, United Kingdom, Oliver and Boyd, 1951.

Anyim, K., Gan, Q. Fault zone exploitation in geothermal reservoirs: Production optimization, permeability evolution and induced seismicity. Advances in Geo-Energy Research, 2020, 4(1): 1-12.

Bagge, M., Hampel, A. Three-dimensional finite-element modelling of coseismic Coulomb stress changes on intracontinental dip-slip faults. Tectonophysics, 2016, 684: 52-62.

Beer, G., Poulsen, B. Efficient numerical modelling of faulted rock using the boundary element method. International Journal of Rock Mechanics and Mining Sciences \& Geomechanics Abstracts, 1994, 31(5): 485-506.

Bletery, Q., Thomas, A., Rempel, A., et al. Imaging shear strength along subduction faults. Geophysical Research Letter, 2017, 44: 11329-11339.

Brady, B. Boundary element methods for mine design. London, University of London, 1979.

Brady, B., Bray, J. The boundary element method for elastic analysis of tabular orebody extraction. International Journal of Rock Mechanics and Mining Sciences \& Geomechanics Abstracts, 1978, 15(1): 29-37.

Cooke, M. Fracture localization along faults with spatially varying friction. Journal of Geophysical Research: Solid Earth, 1997, 102(B10): 22425-22434.

Corkum, A., Damjanac, B., Lam, T. Variation of horizontal in situ stress with depth for long-term performance evaluation of the Deep Geological Repository project access shaft. International Journal of Rock Mechanics and Mining Sciences, 2018, 107: 75-85.

Crouch, S., Starfield, A. Boundary Element Methods in Solid Mechanics. London, United Kingdom, George Allen \& Unwin, 1983.

Dusseault, M., Bruno, M., Barrera, J. Casing shear: Causes, cases, cures. Paper SPE 48864 Presented at SPE International Oil and Gas Conference and Exhibition, Beijing, China, 2-6 November, 1998.

Faraji, M., Rezagholilou, A., Ghanavati, M., et al. Breakouts derived from image logs aid the estimation of maximum horizontal stress: A case study from Perth Basin, Western Australia. Advances in Geo-Energy Research, 2021, 5(1): 8-24.

Fotoohi, K., Mitri, H. Non-linear fault behaviour near underground excavations-A boundary element approach. International Journal for Numerical and Analytical Methods in Geomechanics, 1996, 20(3): 173-190.

Fulton, P., Saffer, D., Bekins, B. A critical evaluation of crustal dehydration as the cause of an overpressured and weak San Andreas Fault. Earth and Planetary Science Letters, 2009, 284(3-4): 447-454.

Gao, F., Kang, H., Li, J. Numerical simulation of fault-slip rock bursts using the distinct element method. Tunnelling and Underground Space Technology, 2021, 110: 103805.

Haimson, B., Lee, C. Hydrofracturing stress determinations at Darlington, Ontario. Canadian Rock Mechanics Symposium (Proceedings), Toronto, Ontario, Canadian Institute of Mining and Metallurgy, 1980, 22: 42-50.

Hawkes, C., McLellan, P. A new model for predicting timedependent failure of shales: Theory and application. Journal of Canadian Petroleum Technology, 1999, 38(12): 49-55.

Hazeghian, M., Soroush, A. Numerical modeling of dipslip faulting through granular soils using DEM. Soil Dynamics and Earthquake Engineering, 2017, 97: 155171.

Henk, A. Stress and strain during fault-controlled lithospheric extension - insights from numerical experiments. Tectonophysics, 2006, 415(1-4): 39-55.

Homberg, C., Hu, J., Angelier, J., et al. Characterization of stress perturbations near major fault zones: Insights from 2-D distinct-element numerical modelling and field studies (Jura mountains). Journal of Structural Geology, 1997, 19(5): 703-718.

Jaeger, J., Cook, N. G. Fundamentals of Rock Mechanics. Oxford, United Kingdom, Wily-Blackwell, 1969.

Kaiser, P. K., Maloney, S. M., Yong, S. Role of large scale heterogeneities on in-situ stress and induced stress fields. Paper Arma 16-571 Presented at 50 ${ }^{\text {th }}$ US Rock Mech/Geomechanics Symposium, Houston, Texas, USA, 26-29 June, 2016.

Kruszewski, M., Hofmann, H., Alvarez, F., et al. Integrated Stress Field Estimation and Implications for Enhanced Geothermal System Development in Acoculco, Mexico. Geothermics, 2021, 89: 101931.

Kuwahara, Y., Choi, J., Cheon, Y., et al. Dependence of earthquake faulting type on fault strike across the Korean Peninsula: Evidence for weak faults and comparison with the Japanese Archipelago. Tectonophysics, 2021, 804: 228757.

Lama, R., Vutukuri, V. Handbook on Mechanical Properties of Rocks: Testing Techniques and Results (Vol. 2). Clausthal-zellerfiel, Germany, Trans Tech Publications, 1978.

Li, K. Numerical analysis of undersea geostress field around fault. Electronic Journal of Geotechnical Engineering, 2015, 20(5): 1887-1896.

Li, L., Aubertin, M., Simon, R. Maximum and minimum horizontal stress evaluation based on the geometry of a single borehole breakout. Paper ARMA-2000-0865 Presented at the $4^{\text {th }}$ North American Rock Mechanics Symposium, Seattle, Washington, USA, 31 July-3 August, 2000.

Lobatskaya, R., Strelchenko, I., Dolgikh, E. Finite-element 3D modeling of stress patterns around a dipping fault. Geoscience Frontiers, 2018, 9(5): 1555-1563. 
Mandl, G. Discontinuous fault zones. Journal of Structural Geology, 1987, 9(1): 105-110.

Pollard, D. D., Segall, P. Theoretical displacement and stresses near fractures in rocks: With application to faults, points, veins, dikes, and solution surfaces, in Fracture Mechanics of Rocks, edited by B. Atkinson, London, United Kingdom, Academic Press, pp. 277-349, 1987.

Ramos, C. C., Daros, C. Stacking sequence optimization of laminated plate structures using the boundary element method. Engineering Analysis with Boundary Elements, 2021, 127: 113-127.

Ritz, E., Mutlu, O., Pollard, D. D. Integrating complementarity into the 2D displacement discontinuity boundary element method to model faults and fractures with frictional contact properties. Computers \& Geosciences, 2012, 45: 304-312.

Rybicki, K. R. Shear stress on a vertical strike-slip fault in a layered medium due to tectonic loading: San Andreas Fault system. International Journal of Rock Mechanics and Mining Sciences \& Geomechanics Abstracts, 1995, 32(4): A152.

Segall, P., Pollard, D. D. Mechanics of discontinuous faults. Journal of Geophysical Research: Atmospheres, 1980, 85(B8): 4337-4350.

Shen, B., Shi, J. A numerical scheme of coupling of fluid flow with three-dimensional fracture propagation. Engineering Analysis with Boundary Elements, 2019, 106: 243-251.

Shi, H., Huang, F., Ma, Z., et al. Mechanical mechanism of fault dislocation based on in situ stress state. Frontiers in Earth Science, 2020, 8: 52.
Streit, J. E. Low frictional strength of upper crustal faults: A model. Journal of Geophysical Research: Solid Earth, 1997, 102(B11): 24619-24626.

Wang, Y., Mi, Q. In-situ stress perturbations and determinations near a fault and their impacts on hydraulic fracturing design. Paper SPE 181779 Presented at SPE Asia Pacific Hydraulic Fracturing Conference, Beijing, China, 24-26 August, 2016.

Wu, K., Olson, J. E. A simplified three-dimensional displacement discontinuity method for multiple fracture simulations. International Journal of Fracture, 2015, 193: 191-204.

Yin, S., Rothenburg, L., Dusseault, M. B. 3D coupled displacement discontinuity method and finite element analysis of reservoir behavior during production in semiinfinite domain. Transport in Porous Media, 2006, 65: 425-441.

Zalohar, J., Vicic, B., Potocnik. M., et al. Precursory stress changes before large earthquakes; on a new physical law for earthquakes. Journal of Structural Geology, 2020, 141: 104208.

Zhang, S., Yin, S. Determination of horizontal in-situ stresses and natural fracture properties from wellbore deformation. International Journal of Oil, Gas and Coal Technology, 2014, 7(1): 1-28.

Zhao, K., Gu, S., Yan, Y., et al. A simple and accurate interpretation method of in situ stress measurement based on rock Kaiser effect and its application. Geofluids, 2018, 2018: 9463439. 